\title{
LES NOYAUX DE BERGMAN ET SZEGÖ POUR DES DOMAINES STRICTEMENT PSEUDO-CONVEXES QUI GÉNÉRALISENT LA BOULE
}

\author{
J.J. LOEB
}

\begin{abstract}
Let be $G$ a complex semi-simple group with a compact miaximal group $K$ and an irreducible holornorphic representation $\rho$ on a finite dimensional space $V$. There exists on $V$ a $K$-invariant Hermitian scalar product. Let be $\Omega$ the intersection of the unit ball ov $V$ with the $G$-orbit of a dominant vector. $\Omega$ is a generalization of the unit ball (case obtained for $C=S L(n, \mathrm{C})$ and $\rho$ the natural representation on $\mathrm{C}^{n}$ ).

We prove that for such manifolds, the Bergman and Szcgö kernels as for the ball are rational fractions of the scalar products and this fractions can be computed explicitely, using invariants of $\rho$. To compute this kernels, one uses a good orthonormal basis related to $\rho$, and then proves that one has a rational fraction, using Schur's orthogonality relations and Weyl's dimensional formula for $V$.
\end{abstract}

\section{Introduction.}

Pour la boule unité de $\mathrm{C}^{n}$ : les noyaux de Szegö et de Bergman sont respectivement donnés (à unc constante multiplicative près) par $(1-<$ $x, y>)^{-n}$ et $(1-\langle x, y\rangle)^{-n-1}$ (ò̀ $<$, $>$ dénotc le produit scalaire herrnitien usuel dans $\mathbf{C}^{n}$ ). Nous donnons ici la gćnéralisation suivante: Soit $G$ un groupe de Lie scmi-simple complexe opérant irreductiblement sur un espace complexe de dimension finie $V$. On munit $V$ d'un produit scalaire hermitien invariant par un sous-groupe compact maximal de $G$ et on considère la varieté $\Omega$ obtenue comme intersection de la boule unité et de l'orbite d'un vecteur dominant. Le résultat que nous allons démontrer est le suivant:

Pour une telle varicté, les noyaux de Berginan ct de Szegö s'expriment comme des fractions rationnelles du produit scalaire. Quand $G$ est simple, le bord de $\Omega$ est strictement pseudo-convexe et on a ainsi une généralistion maturelle du cas de la boule. 
Notations et rappels.

On note $\mathcal{O}(X)$ l'espace des fonctions holomorphes sur une varieté complexe $X$.

Pour un groupe compact $K$, on se donne une famille de représentations irreductibles unitaires $\delta$ sur $E_{\delta}$, inéquivalentes entre elles et décrivant (à equivalence près) toutes les représentations. On note $V_{\delta}$ l'espace engendré par les fonctions $\langle\delta(k) x \mid y\rangle \quad\left(k \in K ; x, y \in E_{\delta}\right.$ et \langle|$>$ dénote un produit scalaire hermitien sur $\left.E_{\delta}\right)$. D'aprés le théorème de Peter-Weyl, on a: $L^{2}(K)=\bigoplus_{\delta} V_{\delta}$ (décomposition hilbertienne). Lorsque $G$ est un groupe réductif complexe et $K$ un sous-groupe compact maximal, la décomposition précédente se complexifie et on a: $\mathcal{O}(G)=\bigoplus_{\delta} W_{\delta}$ où $W_{6}$ est l'espace engendré par les fonctions holomorphes $\langle\delta(g) x \mid y\rangle$ $(g \in G)$. Cette fois cil les convergences sont uniformes sur les compacts de $G$. $W_{\delta}$ est l'espace de la représentation antiholomorphe adjointe de $\delta$. Soit $H$ un sous-groupe complexe fermé de $G$. Notons par $\left(W_{\delta}\right)_{H}$ l'espace des éléments de $W_{\delta}$ qui sont $H$-invariarts à droite. (Ils s'identifient à certains éléments de $\mathcal{O}(G / H))$. On a la décomposition:

$$
\mathcal{O}(G / H)=\bigoplus_{\delta}\left(W_{\delta}\right)_{H}
$$

Ces résultats ont été demontrés par Harish-Chandra dans un cas plus général. Les enoncés qui précédent figurent par exemple dans [B-O].

Les résultats qui précédent s'adaptent immćdiatement au cas d'un ouvert $\Omega$ dans $G / H$ et qui est $K$-invariant. On a alors: (1) $\mathcal{O}(\Omega)=$ $\bigoplus_{\delta}\left(W_{\delta}\right)_{H}$. (Les convergences sont prises ici uniformćrnent sur les compacts de $\Omega$ ).

Soit $m_{\delta}$ la dimerision de l'espace des vecteurs $H$-invariants dans $E_{b}$. Alors $\left(W_{\delta}\right)_{H}$ est en tant que $G$-module une somme directe de $m_{\delta}$ copies de $E_{\delta}$ munies de la structure de module adjoint, de $\left(E_{\delta}, \delta\right)$. En effet, soit $Y_{1}, \ldots, Y_{n}$ une base de $E_{\delta}$, un élément de $V_{\delta}$ s'écrit de façon unique sous la forme: $[\mathrm{WaI}]$

$$
\sum_{i}<\delta(g) X_{i} \mid Y_{i}>
$$

L'invariance par $H$ donne: $\delta(h) X_{i}=X_{i} \quad \forall h \in H$, d'où le résultat.

Dans la suite, nous nous plaçons dans la situation: $G$ semi-simple complexe avec un système de racines positives fixé. On se donne également un poids dorninant $\Lambda$ auquel est associé une représentation $\left(\pi_{\Lambda}, E_{\Lambda}\right)$ muni d'un produit scalaire Hermitien $K$-invariant noté ( 1 ). Soit $v_{\Lambda}$ un vecteur dominant de norme 1 . On note $P_{\mathrm{A}}$ le stabilisateur de $v_{\mathrm{A}}$ qui est contenu 
dans un groupe parabolique $P$. On considère la décomposition (1) dans le cas $H=P_{\Lambda}$. Si $m_{\delta} \neq 0$, alors le poids dominant d'un $\left(W_{\delta}\right)_{H}$ est nécessairement de la forme $n . \Lambda(n \geq 0)$. Dans ce cas $m_{\delta}=1$. $E_{\delta}$ est le sous $G$-module de $E_{\Lambda}^{\otimes n}$ engendré par $v_{\Lambda}^{\otimes n}$. On notera pour la suite $E_{\delta}=E_{n}$ et $\pi_{n}$ la représentation associéc et $\left(W_{\delta}\right)_{H}=W_{n}$. On a:

$$
\mathcal{O}(\Omega)=\bigoplus_{n \geq 0} W_{n}
$$

On choisit par la suite pour $\Omega$ l'image dans $G / P_{\Lambda}$ des $g \in G$ vérifiant: $<g v_{\Lambda} \mid g v_{\Lambda}><1$.

Remarque: La variété $G / P_{\Lambda}$ s'identifie à la $G$-orbite $X$ de $v_{\Lambda}$. Son adhérence dans $E_{\Lambda}$, qui est aussi son enveloppe d'holomorphie, est un cône. Le groupe $\mathrm{C}^{\text {" }}$ opère par homothétie sur l'orbite. L'ouvert $\Omega$ est alors obtenu comme intersection de la boule unité de $E_{\Lambda}$ avec l'orbite, et la décomposition précédente de $O(\Omega)$ peut se lire comme une décomposition en sèrie de Laurent de: $t \rightarrow f(t . x) \quad(x \in X)$ où les termes négatifs n'interviennent pas car $f$ se prolonge à $\bar{X}$. Dans le cas de la boule de $\mathrm{C}^{n}$, on choisit la représentation naturelle de $S L(n, \mathbf{C})$ sur $\mathbf{C}^{n}$ et les $W_{n}$ sont les espaces de polynómes homogènes de degré $n$.]

\section{Les noyaux de Bergman et de Szegö.}

Le noyau de Bergman a été défini pour les variétés dans [We]. En principe, c'est une forme différentielle de type $(m, m)$ où $m$ est la dimension complexe de la varieté $M$. S'il existe sur $M$ une $m$-forme holomorphe $w$ ne s'annulant pas, alors on peut identifier le noyau de Bergman avec une fonction $B(x, y) \in C^{\infty}(X \times X)$ qui est holomorphe en $x$ et antiholomorphe en $y$ et telle que: $\forall f \in L^{2}(X, w \wedge \bar{w}) \cap \mathcal{O}(X)$

$$
f(x)=\int_{X} f(y) B(x, y) w_{y} \wedge \bar{w}_{y} \quad(x \in X)
$$

Ce noyau est alors obtenu par la formule:

$$
B(x, y)=\sum_{n} \Phi_{n}(x) \overline{\Phi_{n}(y)}
$$

où les $\Phi_{n}$ constituent une base orthonormée quelconque de $L^{2}(X, w \wedge \vec{w})$.

Le noyau de Szegö est défni par exemple dans [Kra, (p. 55-57)] pour des domaines bornćs de $\mathrm{C}^{n}$ à bord régulier. Dans notre cas, si on appelle 
$d k$ la mesure de Haar normalisée sur $K$, c'est un noyau $S(x, y)$ continu dans $\Omega \times \bar{\Omega}$, holomorphe en $x$, antiholomorphc en $y$ et tel que:

$$
\forall f \in C(\bar{\Omega}) \cap \mathcal{O}(\Omega): f(x)=\int_{K} f\left(k \cdot x_{0}\right) S\left(x, k x_{0}\right) d k
$$

pour $x \in \Omega$. ( $x_{0}$ élément quelconque de $\partial \Omega$ )

Comme pour le noyau de Bergman, on a: [Kra]

$$
S(x, y)=\sum \Phi_{n}(x) \overline{\Phi_{n}(y)} \quad x, y \in \Omega
$$

où les $\Phi_{n}$ forment une base orthonormée de l'espace de Hilbcrt qui est le complété de l'espace des fonctions de $C(\bar{\Omega}) \cap \mathcal{O}(\Omega)$ muni du produit scalairc:

$$
\langle f, g\rangle=\int_{K} f\left(k x_{0}\right) \overline{g\left(k x_{0}\right)} d k
$$

On va calculer les noyaux de Bergman et Szegö en choisissant des bases $\Phi_{n}$ convenables. Précisons d'abord la forme $w$ que nous choisissons pour $\Omega$ (qui est la restriction d'une forme definie sur tout $G / P_{\Lambda}$ ).

Lemme. Il existe sur $G / P_{\Lambda}$ une forme volume holomorphe $w, G$-invariante et ne s'annulant pas. Cette forme est déterminée à une constante multiplicative près.

Preuve: Comme $G$ est semi-simple, il n'existe pas de caractère non trivial. Par conséquent det $\pi_{\Lambda}(g)=1, \forall g \in G$. Donc sur la $G$-orbite de $v_{\Lambda}$ (qui s'identifie à $G / P_{\Lambda}$ ) il existe une forme holomorphe $G$-invariante et ne s'anmulant pas, obtenue par translation d'une forme en $v_{\Lambda}$ choisie non nulle. L'unicité est trivialc.

Enonçons à présent lc théorème.

Théorème. Les noyaux de Bergman et Szegö sur $\Omega$ sont des fractions rationnelles en $\left(\pi_{\Lambda}\left(g_{1}\right) v_{\Lambda} \mid \pi_{\Lambda}\left(g_{2}\right) v_{\Lambda}\right)$. On peut explicitement calculer ces fractions à partir des invariants de $G$ et de la reqrésentation $\pi_{\Lambda}$.

Preuve du théorème:

On met des points sur les objets quotients.

On utilise les trois outils suivants.

(1) Une formule intégrale [Wal], (p. 177)]. 
Soit $G$ un groupe semi-simple avec une décomposition d'Iwasawa : $G=K A N$. On note $\rho$ la demi-somme des racines associée à la décomposition réele dars $\operatorname{Lie}(G)$ (ou la somme des racines associée à la décomposition complexe dans $L i e(G)$. On a la formule:

$$
\forall f \in C_{c}^{\infty}(G): \int_{G} f(g) d g=\int_{K \times A \times N} f(k a n) e^{2 p(L o g a)} d k d a d n
$$

$(d g, d k, d a, d n$ sont des mesures de Haar respectivement sur $G, K, A, N)$.

On peut utiliser cette formule pour calculer unc mesure $d g$ qui est $G$ invariante sur $G / P_{\Lambda}$. L'algèbre de Lie $\underline{a}$ de $A$ se décompose en somme orthogonale par rapport à la forme de Cartan Killing: $a_{\Lambda} \oplus a_{\Lambda}^{\perp}$, où $a_{\Lambda}$ est. l'annulateur de $\Lambda$. On a $\operatorname{dim} a_{\Lambda}^{\perp}=1$ et il existe donc $H_{0}$ unique dans $a_{\Lambda}^{\frac{1}{\Lambda}}$ tel que: $\Lambda\left(H_{0}\right)=1$. On pose: $2 \rho\left(H_{0}\right)=\alpha$. En utilisant [War], on a:

$$
\forall f \in C_{c}^{\infty}\left(G / P_{\Lambda}\right): \int_{G / P_{\mathrm{\Lambda}}} f(\dot{g}) d \dot{g}=\int_{K \times \mathrm{R}} f\left(k e^{t H_{0}}\right) e^{\alpha t} d k d t
$$

(Dans cette dernière intégrale, on identifie une fonction sur $G / P_{\Lambda}$ avcc une fonction sur $K \times \exp \left(a_{\Lambda}^{\perp}\right)$.)

Pour finir on notera que $d \dot{g}=w \wedge \bar{w}$ (à unc constante multiplicative près).

(2) Les formules d'orthogonalitć [Wal, (p. 29 et 30)].

Si $\delta$ et $\delta^{\prime}$ sont deux représentations irréductibles unitaires de $K$, on a, en notant $<\mid>$ les produits scalaires associés,

$$
\begin{aligned}
& \int\langle\delta(k) X \mid Y\rangle\left\langle\overline{\delta^{\prime}(k) X^{\prime} \mid Y^{\prime}}\right\rangle d k \\
& =\left\{\begin{array}{l}
0 \text { si } \delta \text { et. } \delta^{\prime} \text { sont inéquivalentes. } \\
\frac{1}{d_{\delta}}<X \mid Y>\left\langle\overline{X^{\prime} \mid Y^{\prime}}>\text { si } \delta=\delta^{\prime}\right.
\end{array}\right.
\end{aligned}
$$

(où $d_{\delta}$ est la dimension de la représentation $\delta$ ).

(3) La formule de dimension de H. Weyl [Wal, p. 106].

Pour une représentation $\mu$ de poids dominant $\lambda$ de $G$, la dimension $d_{\mu}$ de la représentation est donnée par la formule:

$$
d_{\mu}=\frac{\prod_{\alpha>0}(\lambda+\rho, \alpha)}{\prod_{\alpha>0}(\rho, \alpha)}
$$

En particulier pour $\lambda=n . \Lambda$, on a:

$$
d_{\mu}=\frac{\prod_{\alpha>0}(n \Lambda+\rho, \alpha)}{\prod_{\alpha>0}(\rho, \alpha)}=T_{\Lambda}(n)
$$


Le point important ici est que $T_{\Lambda}(n)$ est un polynôme en $n$.

Passons à la démonstration du théorème pour le noyau de Bergman que nous noterons $B$.

On remarquera tout d'abord que les $W_{n}$ associés à des représentations inéquivalentes pour $n \neq m$ sont orthogonaux. Cornme $w \wedge \bar{w}=d g=$ $e^{\alpha t} d k d t$ les espaces sont aussi orthogonaux pour $d g$. Pour calculer le noyau de Bergman, il suffit donc de construire des bases orthogonales pour chaque $W_{n}$.

On complète le vecteur $v_{1}=v_{\Lambda}$ en une base orthogonale $v_{1} \ldots v_{n}$ pour $E_{\Lambda}=E_{1}$. Sur $E_{\Lambda}^{\otimes n}$ on choisit un produit scalaire proportionnel à celui provenant du produit tensoriel et normalisé par le fait que $v_{\Lambda}^{\otimes n}$ soit unitaire. On choisit sur le sous module $E_{n}$, muni du produit scalaire induit une base orthonorméc $v_{1, n}=v_{\Lambda}^{\otimes n}, v_{2, n} \ldots, v_{r, n}$.

D'après les relations d'orthogonalité précédentes, les fonctions

$$
\left(\pi_{n}(g) v_{1, n} \mid v_{1, n}\right), \ldots,\left(\pi_{n}(g) v_{1, n} \mid v_{\dot{n}, n}\right)
$$

forment une base orthogonale de $W_{n}$. Il faut normer la base. On a:

$$
\begin{aligned}
& \int_{\Omega}\left|\left(\pi_{n}(\dot{g}) v_{1, n} \mid v_{i, n}\right)\right|^{2} d \dot{g} \\
& =\int_{i<0}\left|\left(\pi_{n}\left(k e^{t H_{0}}\right) v_{1, n} \mid v_{i, n}\right)\right|^{2} e^{\alpha t} d k d t \\
& =\int_{\ell<0} e^{2 n t+\alpha t}\left|\left(\pi_{n}(k) v_{1, n} \mid v_{i, n}\right)\right|^{2} d k d t \\
& \text { (on utilise les normalisations de (1)) } \\
& =\frac{1}{2 n+\alpha} \int\left|\left(\pi_{n}(k) v_{1, n} \mid v_{i, n}\right)\right|^{2} d k \\
& =\frac{1}{(2 n+\alpha) T_{\Lambda}(n)} \text { (en utilisant les relation d'orthogonalité) }
\end{aligned}
$$

Par conséquent $\sqrt{(2 n+\alpha) T_{\Lambda}(n)}\left(\pi_{n}(g) v_{1, n} \mid v_{i, n}\right)$ est une base orthonormćc.

Donc on a:

$$
B\left(\dot{g}_{1}, \dot{g}_{2}\right)=\sum_{n} \sum_{i}(2 n+\alpha) T_{\Lambda}(n)\left(\pi_{n}\left(g_{1}\right) v_{1, n} \mid v_{i, n}\right) \cdot \overline{\left(\pi_{n}\left(g_{2}\right) v_{1, n} \mid v_{i, n}\right)}
$$

où $\dot{g}_{1}, \dot{g}_{2} \in \Omega$.

Remarquons à présent que la $K$-invariance de $w$ implique l'invariance par $K$ (sous-groupe d'automorphisms de $\Omega$ ) de $B$. 
On écrit $g_{1}=k_{1} e^{t_{1} H_{0}} \quad g_{2}=k_{2} e^{t_{2} H_{0}}$ où $k_{1}, k_{2} \in K, \quad t_{1}, t_{2}<0$ On a: $B\left(k_{1} e^{t_{1} H_{0}}, k_{2} e^{t_{2} H_{0}}\right)=B\left(k_{2}^{-1} k_{1} e^{t_{1} H_{0}}, e^{t_{2} H_{0}}\right)$

On a pour $i \neq 1$.

$$
\pi_{n}\left(k_{2}^{-1} k_{1} e^{t_{1} H_{0}} v_{1, n} \mid v_{i, n}\right)\left(\overline{\pi_{n}\left(e^{t_{2} H_{0}}\right) v_{1, n} \mid v_{i, n}}\right)=0
$$

$\operatorname{car} \pi_{n}\left(e^{t_{2} H_{0}}\right) v_{1, n}=e^{n t_{2}} v_{1, n}$ et pour $i=1$, la même expression est egale à: $e^{n\left(t_{1}+t_{2}\right)}\left(\pi_{n}\left(k_{2}^{-1} k_{1}\right) v_{1, n} \mid v_{1, n}\right)$ Par conséquent:

$$
\begin{aligned}
B\left(\dot{g}_{1}, \dot{g}_{2}\right) & =\sum_{n} e^{n\left(t_{1}+t_{2}\right)}\left(\pi_{\pi_{l}}\left(k_{2}^{-1} k_{1}\right) v_{1, n} \mid v_{1, n}\right) \cdot(2 n+\alpha) T_{\Lambda}(n) \\
& =\sum_{n}\left(\pi_{n}\left(k_{1}\right) e^{n t_{1}} \cdot v_{1, n} \mid \pi_{n}\left(k_{2}\right) e^{n t_{2}} \cdot v_{1, n}\right) \cdot(2 n+\alpha) T_{\Lambda}(n) \\
& =\sum_{n}\left(\pi_{n}\left(\dot{g}_{1}\right) v_{1, n} \mid \pi_{n}\left(\dot{g}_{2}\right) v_{1_{2} n}\right) \\
& =\sum_{n}(2 n+\alpha) T_{\Lambda}(n)\left(\pi_{\Lambda}\left(\dot{g}_{1}\right) v_{\Lambda} \mid \pi_{\Lambda}\left(\dot{g}_{2}\right) v_{\Lambda}\right)^{n}
\end{aligned}
$$

(car $v_{1, n}=v_{\Lambda}^{\otimes n}$ et $v_{1, n}$ de norme 1).

Posons $R_{1}(x)=\sum_{n \geq 0}(2 n+\alpha) T_{\Lambda}(n) x^{n}$. C'est une fraction rationnelle en $x$ car ses coefficients sont des polynômes en $n$. (On écrit $(2 n+\alpha) T_{\Lambda}(n)$ sous la forme $a_{0}+a_{1} n+a_{2} n(n+1)+\cdots$ et on a donc $R(x)$ en fonction de $\frac{1}{1-x}, \frac{1}{(1-x)^{2}}$ etc...). Ceci finit la démonstration pour le noyau de Bergman.

De la même façon, le noyau de Szegö est obtem à partir de la fraction rationelle $R_{2}(x)=\sum_{n \geq 0} T_{\Lambda}(n) x^{n}$

Remarques finales. 1) Pour $G$ simple, $\partial \Omega$ est. strictemont pseudoconvexe $[\mathbf{R o}]$.

2) Dans le développement asymptotique de $B(\dot{g}, \dot{g})$ il n'y a pas de terme en logarithme. Rappelons que pour un domaine borné $X$ strictement pseudo-convexe de $\mathbf{C}^{n}$, Ramadanov conjecture que l'absencc de terme en logarithme implique que $X$ est équivalent à la boule [Ra]. L'exemple de $\Omega$ montre que cettc conjecture ne s'étend pas aux domaines strictement. pseudo-convexes dans des espaces de Stein. (L'enveloppe d'holomorphie de $\Omega$ est le cône $\overline{G . v_{\Lambda}}$ (voir remarque 1 ).)

\section{References}

[B-O] BARTH-OTTE, Invariante holomorphe funktionen auf reduktive Liegruppen, Math. Ann. 201 (1973). 
\{Kra\} KrAxTz, Function theory of several complex variables.

[Ra] Ramadanov, A characterisation of the balls in $\mathbf{C}^{n}$ by means of the Bergman kerncl, Compte rendu de l'academic bulgare des sciences tome 34,7 (1981).

[Ro] Rossi, Homogcous strongly pseudo-convex surfaces, Rice studies 59 (1973).

[Wal] WALLACH, Harmonic analysis on homogeneous spaces.

[War] WaRner, Harmonic analysis on semi-simple Lie groups.

[We] WeIl, Variètés kähleriennes.

Département de Mat. Iónyetiques

Faculté des Sciencess

Universilé d'Angers

2, Boulevard Lavoisier

49045 Angers

FIRANCE

Rebut el 12 de Desembre de 1990 